\title{
Tagungsbericht
}

\author{
Denise Siemer
}

\section{"Violent Times, Rising Protests. Structures, Experiences and Feelings." Fachtagung der Schweizer Gesellschaft für Geschlechterforschung}

\section{2. und 13. September 2019 in Bern}

Gewalt in ihren zahlreichen Erscheinungsformen und Wirkmächtigkeiten durchzieht unsere Gesellschaften und Lebenswirklichkeiten. In global erstarkenden rassistischen, sexistischen, transund homofeindlichen Kontexten findet sie neue Wege, sich in Körper und Subjekte, Alltag und Strukturen einzuschreiben. Gleichzeitig werden auch Proteste, Widerstand und Bündnisse gegen Gewalt lauter und zahlreicher. Dementsprechend thematisierte die Fachtagung der Schweizer Gesellschaft für Geschlechterforschung (SGGF/ SSEG) am 12. und 13. September 2019 an der Universität Bern sowohl Formen von Gewalt als auch des Widerstandes gegen sie sowie Fragen des (Über)Lebens in Kontexten der Gewalt. Nicht zuletzt ging es auch um die Frage, in welchem Verhältnis die (feministische) Wissenschaft zu einer gewaltvollen Gesellschaft steht.

Die zwei Keynotes der Tagung wurden von Jack Halberstam und Noémi Michel gehalten. Beide beschäftigten sich zentral mit den Möglichkeiten der Kritik von Demokratie und mit der Frage eines neuen, erweiterten Demokratieverständnisses aus feministischer Perspektive.
Jack Halberstam sprach sich in seinem Vortrag mit dem Titel "Destitution, Dereliction, Disorder and Dispossession « für eine radikale und militante Dekonstruktion der Verhältnisse aus. Er bezog sich dazu auf Aktionen feministischen Widerstands der 1970er Jahre und deren Aufnahme in Kunst und Literatur. In Werken, wie z.B. Ursula LeGuins The Dispossesed (1974), im künstlerisch-architektonischen Projekt Nothing Works von Gordon Matta Clark, in den Shooting Paintings von Niki de Saint Phalle oder auch in SciFi Filmen wie Born in Flames von Lizzie Borden ließen sich, so Halberstam, relevante und aktualisierbare Utopien finden, die eine radikale Dekonstruktion der Verhältnisse vornehmen und neoliberale Deutungen von agency und Fortschritt ablehnen. Im Hinblick auf Gewalt werde hier ein Verhältnis von feministischer Theorie und Praxis sichtbar, das von Widerstand und Militanz zeuge und in dem - anders als heute - Vulnerabilität und "victimhood" von Frauen, queeren und nichtbinären Subjekten eine weniger zentrale Bedeutung erhielten.

Noémi Michel sprach in ihrer Keynote mit dem Titel "Unsustainable Inclusion: A black feminist critique of 
democracy« über die Widersprüche demokratischer Inklusion und Partizipation. Sie bezog sich dabei vor allem auf die Erfahrungen von schwarzen Frauen im universitären und öffentlichen Kontexten in der Schweiz. Die Stimmen schwarzer Subjekte würden hier, obwohl zahlreich vertreten, und trotz ihres kritischen und emanzipatorischen Potentials, nicht gehört. Hier zeige sich, dass responsive Prozesse in einer hegemonialen Welt, in der eine kritische, rassifizierte und marginalisierte Stimme systemisch rassistische und patriarchale Augen und Ohren adressiere, nicht funktionierten. Statt die Stimmen schwarzer Subjekte anzuhören, erschienen dem hegemonialen Auge und Ohr markierte Subjekte, die als repräsentabel gelten: nicht kritisch, sondern zufrieden und dankbar für ihre Inklusion in eine hegemoniale Öffentlichkeit. Noémi Michel illustrierte die (Ir)Responsivität sowohl marginalisierter als auch markierter Stimmen und Bilder zum einen mit der Kontroverse um ein rassistisches Wahlkampfposter in der Schweiz, auf dem ein schwarzes Schaf von mehreren weißen Schafen von einer Weide gedrängt wird und eine Bildunterschrift trägt, die für mehr Sicherheit plädiert. Obwohl eine Vielzahl schwarzer Bürger ${ }^{\star}$ innen das Plakat sofort schärfstens kritisierten, wurde es erst aus dem Verkehr gezogen, nachdem auch weiße Politiker^innen dazu kritisch Stellung bezogen. Zum anderen typisierte die Referentin Markierungen der Inklusion und $\mathrm{Re}$ präsentation im akademischen Feld, indem sie eine aus Erfahrungen schwarzer Frauen verdichtete Fallgeschichte von V. erzählte: Hier wird eine junge schwarze Frau gebeten, die Universität bei öffentlichen Vorträgen zu repräsentieren und sich auf einem Poster der Universität ablichten zu lassen. Als V. sich in universitären Gremien einbringt und Kritik an universitären Strukturen äußert, bleibt ihre Stimme ungehört und sie resümiert: »I should be there, but not be part. They want my image, but not me.«

\section{Transnationale Gewaltstrukturen}

Neben den Keynotes gab es sechs "Tracks", die je vier zeitgleich stattfindende "Panels« umfassten. Ich gebe im Folgenden einen Einblick in ausgewählte Panels, in denen globale und lokale Gewaltstrukturen und deren transnationale Verflechtungen verhandelt wurden und die neben der Analyse von Macht- und Gewaltverhältnissen auch Möglichkeiten des Widerstands und der Solidarität thematisierten.

In dem Panel »Organized Fear: State Intervention and Power Reproduction" sprach auch Isis Giraldo. Sie stellte ihre Arbeit zu den sogenannten "Falsos Positivos" in Kolumbien vor, bei denen mehrere Tausend junge Männer hingerichtet und der Öffentlichkeit nachträglich als »Guerillakämpfer« präsentiert wurden. Obwohl umfängliche Informationen über die Massenhinrichtung und die Falschdarstellung zur Verfügung standen, wurde die Tat nie aufgeklärt. Die fehlende Empörung der kolumbianischen Öffentlichkeit erklärte Giraldo mit einer "pedagogy of curelty«, die erneut patriarchale und nationalistische Ideologien ins Zentrum rückt. In der Perspektive solcher Ideologien werden die ermordeten Männer, die überwiegend arm waren und aus der Unterschicht kamen, in das 
Licht kapitalistischer Verwertbarkeitslogik gestellt, als sunproduktiv und als Bürger zweiten Klasse deklariert. Durch die Einübung eines Blicks auf die Ermordeten als vermeintlich unproduktiv und als jenseits von "fine citizenship«, etablierte sich in der bürgerlichen Öffentlichkeit die Einstellung, die Morde unwidersprochen hinzunehmen. Damit wurde zugleich der Deutung Anerkennung geschaffen, Mord im Namen des Fortschritts zu rechtfertigen.

In dem Panel »Feminist Activism and the Politics of Protest« stellte Cecile Heim ihre Forschungsabeit vor. Sie befasste sich mit kultureller Gewalt und künstlerischen Praktiken des Widerstands. Als Veranschaulichungsbeispiel diente ihr der kanadische »Indian Act«, ein paternalistisches Gesetz, das sämtliche Statusfragen indigener kanadischer Subjekte verhandelt und auch die Etablierung sogenannter "Residence Schools" begründete. Dies sind Schulen innerhalb indigener Reservate, in denen im Zuge systematischer und unter Anwendung von körperlicher und psychischer Gewalt Enttraditionalisierungserziehung auch Tausende Kinder getötet wurden. 25\% aller Kinder, die eine Residence School besuchten, starben durch das Personal; an Misshandlungen, Vergewaltigungen, Krankheiten oder Unterernährung. Heim konstatierte ein intergenerationelles Trauma innerhalb der indigenen community Kanadas. Anknüpfungspunkte zur Bearbeitung und Überwindung dieses Traumas veranschaulichte die Referentin am Beispiel des Films "Rhymes for Young Ghoules" von Jeff Barnaby. Der Film erzählt die Geschichte eines indigenen weiblichen
Teenagers, die sich nach dem Tod ihres Vaters und der Übernahme seines Drogenhandels bei einem korrupten Schulleiter aus einer Residence School freikauft und ihre Identität mit Hilfe von kulturellem Wissen der Indigenen und traditionellem Storytelling neu ausrichtet, beispielsweise wenn sie von ihrer Großmutter alte Geschichten hört oder in kunstvollen Zeichnungen ihrer verstorbenen Mutter blättert. Der Wissenstransfer hilft der Protagonistin, sich gegen koloniale und epistemologische Gewalt effektive zur Wehr zu setzen und emanzipatorischen Widerstand zu praktizieren.

Im Panel zu »Racial Profiling. Intersektionale Perspektiven« kamen auch aktivistische Akteur^innen zu Wort. So stellte unter anderem das Kollektiv Rassismus vor Gericht seine Arbeit vor. Die Gruppe betreibt kritische Prozessbeobachtung und -protokollierung, um Mechanismen einer weißen und rassistischen Justiz sichtbar zu machen und sich mit Opfern eben dieser Justiz solidarisch zu zeigen. Vorgestellt wurden Teile eines Prozessprotokolls des Falles von Winston A. und seiner Verhandlung 2018 in Zürich. A. war im Oktober 2009 Opfer einer rassistischen Polizeikontrolle geworden und klagte, nach einer gewaltvollen und lebensbedrohlichen Eskalation seitens der Polizei, gegen drei weiße Polizist $\star_{i n n e n .}$ Das Protokoll illustriert die "whiteness" der gerichtlichen Gesamtsituation. Verhandelt wurde lediglich die Glaubwürdigkeit der Parteien, wobei die Erfahrungen von Winston A. individualisiert und die von ihm erfahrene Gewalt als subjektives Empfinden deklassiert wurden. Die strukturell rassistischen 
Dimensionen der Tat hingegen fanden weder Erwähnung noch Anerkennung.

Im Panel »Recht als Gewalt - Recht gegen Gewalt« ging es um das Gewaltpotenzial, das im Recht selbst verankert ist, wie auch um die Möglichkeiten, Gewalt mittels Recht einzudämmen. Hier sprachen Nula Frei und Anne-Laurence Graf über die IstanbulKonvention, eine 2011 im Europarat verabschiedete Konvention zur Vermeidung und Ahndung von Gewalt gegen Frauen und häuslicher Gewalt, und deren Umsetzung in der Schweiz. Die Referentinnen würdigten die Konvention als das erste internationale Rechtsdokument, das Gewalt gegen Frauen als Diskriminierung verhandelt. Sie biete international zum ersten Mal die Möglichkeit, strukturelle Gewalt gegen Frauen zu verurteilen. Auch erkenne die Konvention unterschiedliche statusbezogene Vulnerabilitäten von Subjekten im globalen und transnationalen Raum an. Allerdings stehe auch in der rechtspolitischen Praxis der Schweiz die Einbeziehung von u.a. Trans`frauen weiterhin aus. AuBerdem verpflichte die Konvention nicht zu Maßnahmen in Fällen struktureller Gewalt. So zeige sich die Schweiz bisher nicht bereit, Formen sozio-kultureller Gewalt zu ahnden, z.B. die im Ausland an in der Schweiz lebenden Frauen begangene Genitalverstümmlungen.

Insgesamt vermittelten die Beiträge und Debatten der Tagung diverse Perspektiven auf Gewalt, stellten vielfältige Konzepte von Gewalt miteinander in Beziehung und reflektierten die Möglichkeiten Widerstand oder gar Überwindung von Gewalt. Dabei wurde deutlich, dass es die eine feministische Antwort darauf, was Gewalt ist und wie sie wirkt oder wie man sich ihr widersetzen kann, nicht gibt. Vielmehr machten die zwei Tage in Bern die Produktivität intersektionaler Perspektiven deutlich. 